\title{
AmpC $\beta$-lactamase Production in Pseudomonas aeruginosa: A Threat
}

\author{
Arti Agrawal ${ }^{1}$, Namita Srivastava ${ }^{1}$, Dharmendra Kumar ${ }^{2 *}$, Rashmi $^{2}$ and Neha Bhati ${ }^{2}$ \\ ${ }^{1}$ Department of Microbiology, Sarojini Naidu Medical College, Agra (Uttar Pradesh), India \\ ${ }^{2}$ Department of Microbiology, Chhatrapati Shahujii Maharaj University, \\ Kanpur (Uttar Pradesh), India \\ *Corresponding author
}

\begin{tabular}{|c|c|}
\hline & A B S T R A C T \\
\hline & \multirow{8}{*}{$\begin{array}{l}\text { Pseudomonas aeruginosa is responsible for the devastating nosocomial infections. Class C } \\
\text { of cephalosporinase (AmpC } \beta \text {-lactamases) is important cause of multiple } \beta \text {-lactam } \\
\text { resistance in P. aeruginosa. The aim of this study is to detect the AmpC } \beta \text {-lactamase } \\
\text { production and Antimicrobial susceptibility pattern in Pseudomonas aeruginosa isolated } \\
\text { from clinical samples in Tertiary health care Hospital. This study was conducted over a } \\
\text { period of January (2016) to July (2016) in department of Microbiology, Sarojini Naidu } \\
\text { Medical College, Agra (Uttar Pradesh), India. The organisms were identified on the basis } \\
\text { of their culture characteristics and battery of Biochemical tests. Antimicrobial } \\
\text { susceptibility of all the isolates was performed by the Kirby- Bauer disc-diffusion method. } \\
\text { AmpC } \beta \text {-lactamase production was determined by the modified 3D test. } 159 \text { isolates of } P \text {. } \\
\text { aeruginosa were obtained from different specimens. Maximum numbers of } P \text {. aeruginosa } \\
\text { isolates were resistant to Piperacillin followed by Gentamycin, Cefepime and Ceftazidime. } \\
\text { While most isolates were sensitive to Polymyxin-B, Colistin, Amikacin, Imipenem, and } \\
\text { Meropenem. A high prevalence of AmpC } \beta \text {-lactamases production was observed amongst } \\
\text { the isolates of } P \text {. aeruginosa as compared to other studies. Isolates of AmpC } \beta \text {-lactamases } \\
\text { producing Pseudomonas aeruginosa have been continuously reported globally with some } \\
\text { disparity in the rates of resistance. }\end{array}$} \\
\hline Keywords & \\
\hline $\begin{array}{l}\text { Pseudomonas } \\
\text { aeruginosa, AmpC } \\
\beta \text {-lactamases, }\end{array}$ & \\
\hline $\begin{array}{l}\text { AmpC } \beta \text {-lactamase } \\
\text { Screening and }\end{array}$ & \\
\hline $\begin{array}{l}\text { Confirmatory 3D } \\
\text { test. }\end{array}$ & \\
\hline Article Info & \\
\hline $\begin{array}{l}\text { Accepted: } \\
17 \text { October } 2017 \\
\text { Available Online: } \\
10 \text { December } 2017\end{array}$ & \\
\hline & \\
\hline
\end{tabular}

\section{Introduction}

Pseudomonas aeruginosa is an opportunistic gram-negative bacterium that causes of 9$10 \%$ of nosocomial infections (1). Despite advances in medical and surgical care and introduction of wide variety of antimicrobial agents with anti-pseudomonal activities, life threatening infection caused by Pseudomonas aeruginosa continue to cause complication (2). Antibiotic resistance is a major threat in contemporary medicine. Beta-lactamases enzymes are one of the major mechanisms of resistance to $\beta$-lactam antibiotics in
Pseudomonas aeruginosa $(3,4)$. AmpC betalactamases that belongs to molecular class $\mathrm{C}$, are bacterial enzymes that hydrolyse 3rd generation extended spectrum cephalosporins and cephamycins, monobactams and carbepenems endangering resistance to these categories of antibiotic. Two features differentiate AmpC $\beta$-lactamases from other $\beta$-lactamases such as Extended spectrum $\beta$ lactamases (ESBLs): their resistance to ESBLs inhibitors such as clavulanate and their ability to hydrolyze cephamycins such as 
cefoxitin and cefotaxime $(5,6)$. AmpC in $P$. aeruginosa usually are encoded by the chromosomal genes and expressed constitutively at a low level (5). Mutations in ampC may lead to overproduction of AmpC $\beta$-lactamases by some $P$. aeruginosa isolates (5). AmpC overproduction not only causes resistance to cephalosporins, cephamycins and monobactams but also is responsible for resistance to carbapenems (3, 5). Characteristically, AmpC $\beta$-lactamases are known to mask ESBL production in organisms harboring both AmpC and ESBL, and they are poorly inhibited by Clavulanic acid. AmpC enzymes are also inhibited by $4^{\text {th }}$ generation cephalosporins (such as Cefepime) and the Carbapenems (IPM and MRP) and there production can either be caused by mutation or as a result of an inducing agent. Induction of resistance to $3^{\text {rd }}$ generation cephalosporins after exposure to these agents complicates treatment options and that infections caused by AmpC $\beta$-lactamase producing organisms can successfully be treated with Carbapenems $(7,8)$. Cefepime a $4^{\text {th }}$ generation cephalosporins with broader spectrum activity compared to Ceftriaxone (9, 10). Cefepime, with its dipolar charge, has the advantage of penetrating bacterial outer membranes more rapidly than $3^{\text {rd }}$ generation cephalosporins, readily reaching its target and avoiding $\quad \beta$-lactamase inactivation. Additionally, Cefepime has a reduced affinity for $\beta$-lactamases and also appears to be a poor inducer of AmpC $\beta$-lactamases $(11,12)$. The therapeutic effectiveness of Cefepime compared with Carbapenems has not been previously evaluated. Infections caused by AmpC $\beta$-lactamases producing organisms treated with Cefepime to those of patients treated with Meropenem.

Pseudomonas aeruginosa is a nosocomial pathogen and it exploits some break in the host defences to imitate an infection. Pseudomonas causes urinary tract infection
(UTI), respiratory system infections, dermatitis soft tissue infections, bacteraemia, bone and joint infections, gastrointestinal infections and variety of systemic infections. It is particularly important in patients with serve burns and in cancer and AIDS patients who are immunosupressed. They are mostly prevalent in moist environment. Hospital environments, especially ICUs are common habitat for $P$. aeruginosa. Pseudomonas is one of the most vigorous, fast swimming bacteria seen in hay infusions and pond water sample consist of acetate as a source of Carbon and $\mathrm{NH}_{4} \mathrm{SO}_{3}$ (ammonium sulphate) as a source of Nitrogen.

The metabolic versatility for which Pseudomonas are so renowned. It can tolerant to wide variety of physical conditions, including temperature resistant to high concentration of salt and days, weak antiseptics and many commonly used antibiotics. It has predilection for growth in moist environment which is probably a reflection of its natural existence in soil in water. Pseudomonas aeruginosa was named among the top 10 infectious diseases in the world, not only for its infectious qualities but survivability as an antibiotic resistant bacterium.

\section{Materials and Methods}

The present study was conducted at the Department of Microbiology, Sarojini Naidu Medical College, Agra (Uttar Pradesh), India during the period from January 2016 to July 2016. Samples received from various ICU units, wards of Ear, Ophthalmology, NICU, PICU, Gynae and Obstetrics OPD (outpatient department), NPB and EDpedia were inoculated on Nutrient agar, Blood agar and MacConkey agar, CLED media plates and incubated overnight at $37^{\circ} \mathrm{C}$ aerobically. Bacterial pathogens were identified by conventional biochemical methods according 
to standard microbiological techniques. Pseudomonas aeruginosa was identified by colonial morphology, a positive oxidase reaction, pyocyanin production, motility. Colonies which displayed a positive oxidase test were further subjected to biochemical reactions.

\section{Antimicrobial susceptibility testing}

It was performed on Muellar-Hinton agar (HiMedia, India) by Kirby Bauer disk diffusion method by using antibiotic discs of Polymyxin-B (PB), Colistin (CL), Meropenem (MRP), Amikacin (AK), Imipenem (IPM), Piperacillin/Tazobactam (PIT), Levofloxacin (LE), Aztreonam (AT), Cefepime (CPM), Ceftazidime (CAZ), Gentamycin (GEN), Piperacillin (PI).

\section{Screening for AmpC production}

The $P$. aeruginosa isolates were tested for AmpC production using the cefoxitin disc (30 $\mu \mathrm{g})$ (Becton, Dickinson and Company, USA) (13). Bacterial suspension of 0.5 McFarland density of the test isolate was prepared and inoculated onto the surface of the MuellerHinton agar.

The cefoxitin antibiotic disc was placed on the inoculated agar and incubated overnight at $37^{\circ} \mathrm{C}$. Isolates that yielded zone diameters of less than $18 \mathrm{~mm}$ or resistance were considered positive for AmpC beta-lactamase production in the screening test (13). A previously known AmpC producing $P$. aeruginosa isolate and $E$. coli ATCC 11775 were used as controls.

\section{AmpC confirmatory test}

The modified 3D test was used to confirm the production of both inducible and noninducible AmpC $\beta$-lactamases (13)

Procedure - Overnight culture on Muller Hilton Agar was transferred to sterile micro- centrifuge tube containing peptone water and then pelleted by at 3000 rpm by centrifugation for 15 minutes. The supernatant was decanted and fresh peptone water was added and the bacterial suspensions were repeatedly frozen and thawed about 10 times to obtain crude AmpC enzyme extract.

Mueller-Hinton agar surface was inoculated with E. coli ATCC 11775 to produce a lawn culture and Cefoxitin $(30 \mu \mathrm{g})$ disc was placed at the centre of the plate. On the agar, three linear slits about three centimeters $(3 \mathrm{~cm})$ long were made three millimeters $(3 \mathrm{~mm})$ away from the Cefoxitin disc using a sterile surgical blade.

A small circular well was made on each slits 5 $\mathrm{mm}$ from the Cefoxitin disc by stabbing the agar with the tip of a sterile Pasteur pipette. One of the wells was filled with the enzyme extracted from the test organism.

Each of the remaining wells was filled with extracts from the positive control (previously known $P$. aeruginosa isolate) and the third well filled with the negative control (E. coli ATCC 11775).

They were then incubated at $37^{\circ} \mathrm{C}$ overnight. After incubation, isolates that showed clear distortion of zone of inhibition around Cefoxitin discs were confirmed as AmpC producers. The isolates that produced no zones of distortion were AmpC nonproducers. (14)

\section{Results and Discussion}

Total $159 P$. aeruginosa were isolated from different types of specimen from different age groups i.e., Pus (46 case), Pleural fluid (34case), Sputum (30 case), Gastric aspirate (11 case), Urine (9 case), Blood (9 case), Ear swab (3case), ET tip (3 case), Catheter tip (2case), Conjunctival swab (1case), IV line (1 case), Central line (1case). 
On the basis of AmpC $\beta$-lactamases production

Total 73 cases $(46.00 \%)$ are recorded in AmpC $\beta$-lactamases production which is resistance to broad spectrum Cephalosporins e.g., Ceftazidime, Cefepime.

$P$. aeruginosa is a major cause of nosocomial infections worldwide and ranking second among the gram-negative pathogens reported to the NNIS (National Nosocomial Infection Surveillance System) (15, 16 and 17).

In this study, $P$. aeruginosa are isolated and identified from various clinical samples and their AmpC $\beta$ - lactamase, patterns are determined.

Antibiotic susceptibility patterns serve as a useful guideline for choosing the appropriate antibiotics. There is no standard definition of MDR in Pseudomonas aeruginosa till now.

On comparison we found that some important and big difference in results in other studies.

In the present study, high prevalence of AmpC production (46.00\%) among the isolates as comparable with Rodrigues et al., (18) (26.5\%), Bhattacharjee et al., (19) (22\%).
AmpC $\beta$-lactamase enzymes are inducible and can be expressed at high levels by mutation. Over expression confers resistance to broad spectrum cephalosporins including Cefotaxime, Ceftazidime, and Ceftriaxone and is a problem especially in nosocomial infections. $\beta$-lactam / $\beta$-lactamase inhibitor combinations and most cephalosporins and Penicillins should be avoided because of in vitro resistance, the potential for $\mathrm{AmpC}$ induction or selection of high-enzyme-level mutants, and documented poor clinical outcomes with Ceftazidime, Cefotaxime.

The susceptibility pattern of clinical isolates of $P$. aeruginosa showed higher resistant to Piperacillin $87.92 \%$ which was $90 \%$ in Spain (20), $88 \%$ in Italy (21), $86 \%$ in America.

Resistant to Carbapenems, Imipenem 21.00\% and Meropenem $20.50 \%$ is almost similar as in Spain resistance; $14 \%$ in Spain (20), 19.3\% in Italy (21), and $32 \%$ in Saudi Arabia (22). The National Nosocomial Infection Surveillance System (NNIS) reported the incidence of IPM resistance as $18.5 \%$ among isolates of $P$. aeruginosa (23). The MRP Yearly Susceptibility Test Information Collection (MYSTIC) study group reported an incidence of $19 \%$ in 10 medical centers (24) (Fig. 1 and 2).

Fig.1 Antimicrobial susceptibility pattern by disk diffusion method

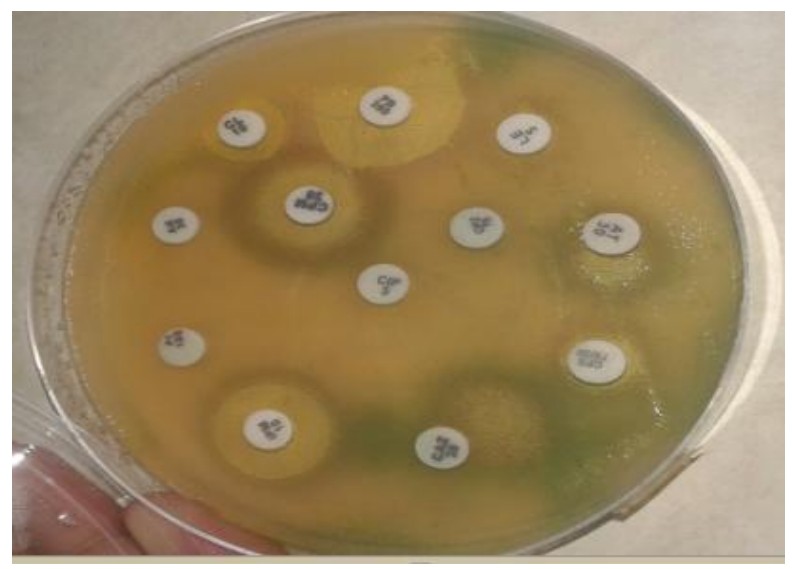


Fig.2 AmpC confirmatory test by 3D test

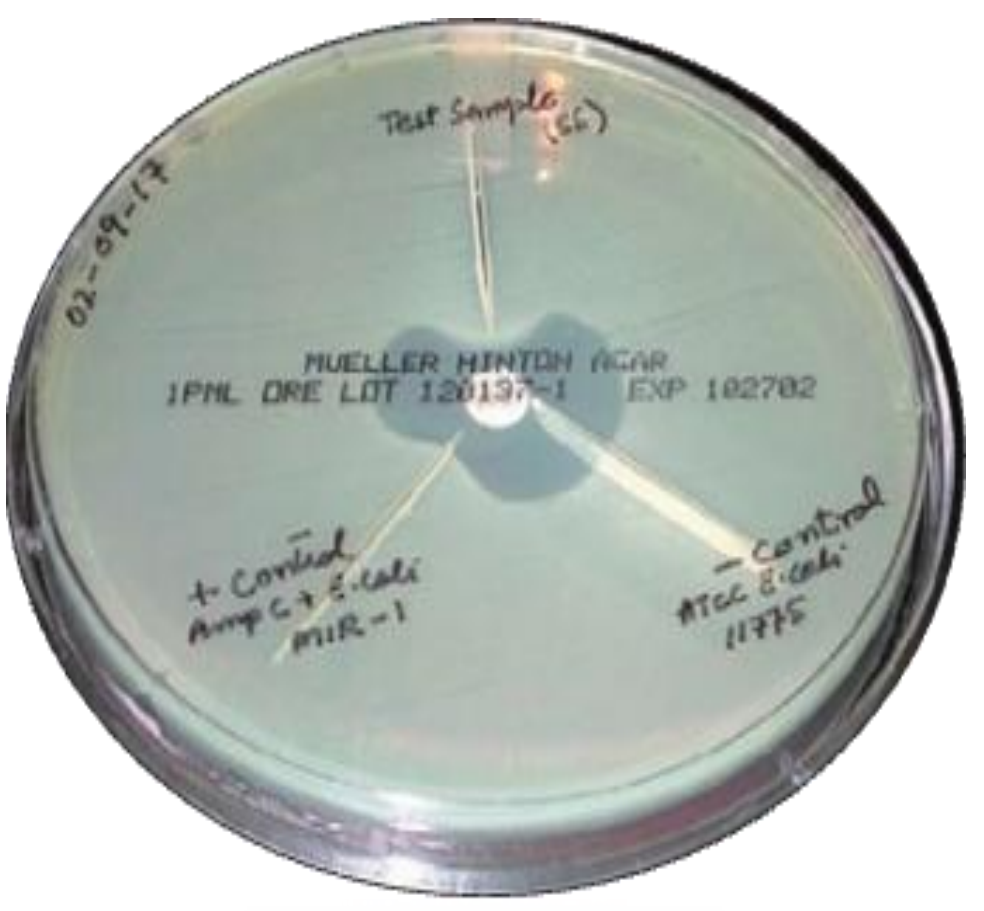

Gender distribution of cases of Pseudomonas aeruginosa

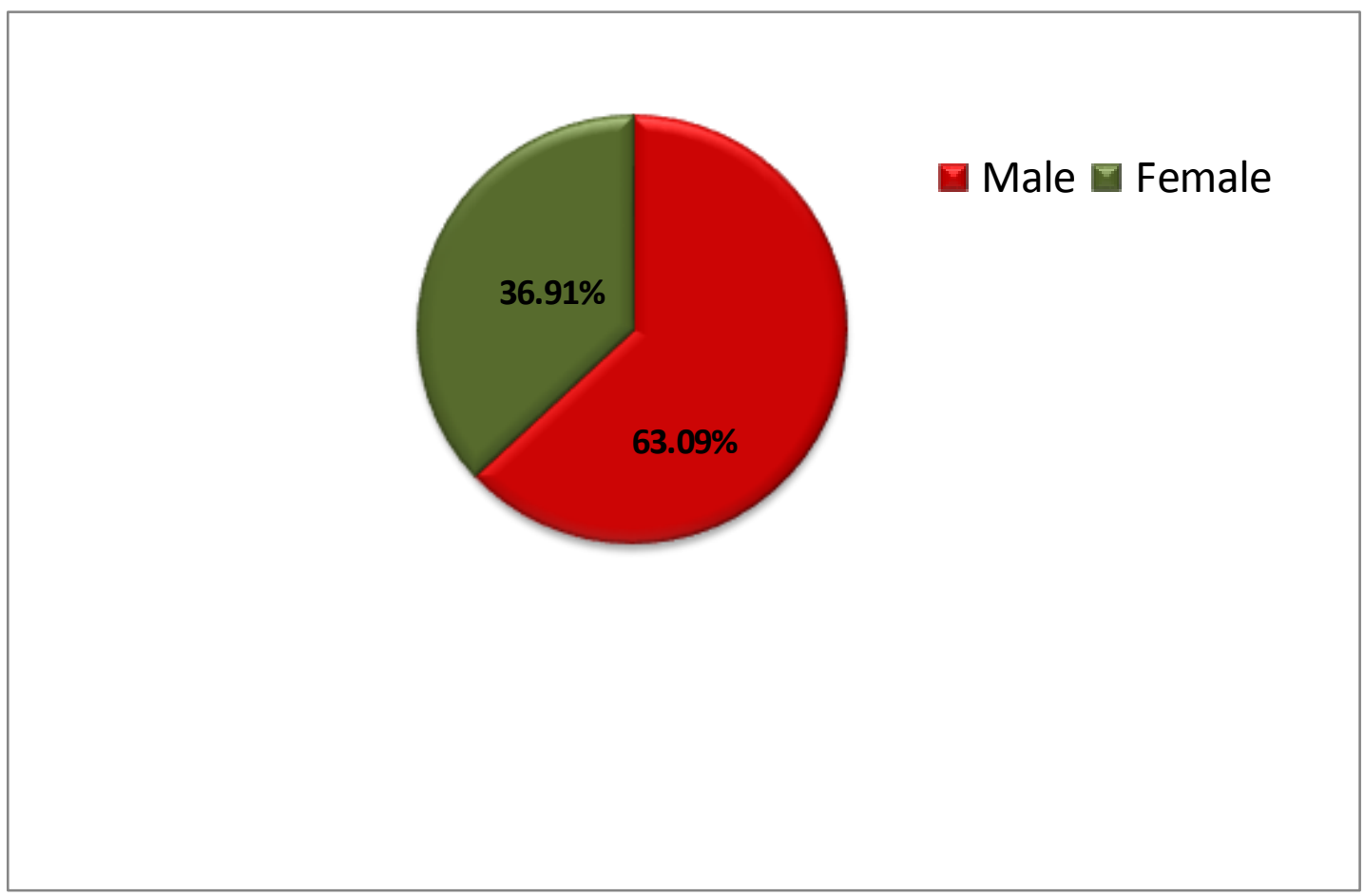

Male patients - 94 cases (63.09\%), Female patients - 55 cases (36.91\%) Neonates - 10 cases Pseudomonas aeruginosa infection is more common in, Males than Female patients. 


\section{Graphical distribution of Antimicrobial susceptibility pattern of Pseudomonas aeruginosa isolated from different clinical samples}
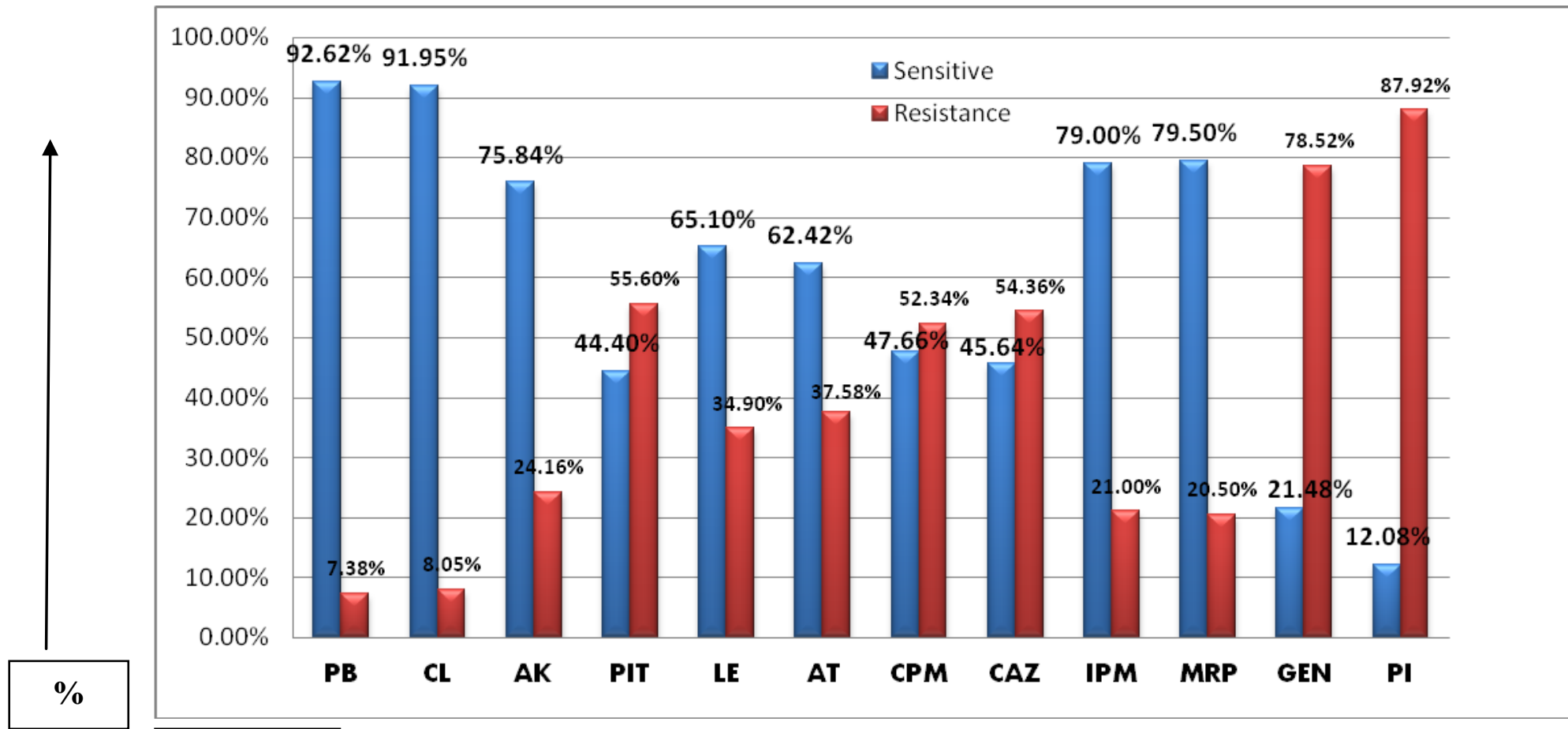

\section{Antibiotic}

People may cause easily nosocomial infection worldwide due to poor hygiene. Male patients are predominant than female which is the likely case in earlier reports. Possibly, it may be due to high incidence of road traffic accidents among males, leading to hospitalization there by high incidence of $P$. aeruginosa infections through catheterization. In hospitals, where the most serious infections occur, Pseudomonal infections can be spread on the hands of healthcare workers or by equipment that gets contaminated and is not properly cleaned.

This study shows that the clinical isolates of Pseudomonas aeruginosa are becoming resistant to commonly used antibiotics and gaining resistance to newer antibiotics. Ongoing studies on current antimicrobial resistance profiles of $P$. aeruginosa are essential to find out the susceptibilities of this pathogen against in any health care facility. This would help the physicians to optimize the current therapeutic treatment options. Although previous studies have indicated that $3^{\text {rd }}$ generation cephalosporins appears to be suboptimal choices for treating infections caused by pathogens producing AmpC $\beta$ lactamases, the role of Cefepime has been unsettled and many experts recommend resorting to Carbapenem therapy, which appear to have both excellent in vitro and vivo activity against these organism $(25,26,27,28,29,30,31)$.

This study shows that the clinical isolates of Pseudomonas aeruginosa are becoming resistant to commonly used antibiotics and gaining resistance to newer antibiotics. 
The antimicrobial agents are losing their efficiency because of the spread of resistant organisms due to indiscriminate use of antibiotics, lack of awareness, patient noncompliance and unhygienic conditions.

Prevalence of AmpC $\beta$-lactamases producing clinical isolates of Pseudomonas aeruginosa have been continuously reported globally with some disparity in the rates of resistance.

According to this study, Polymyxin-B, Colistin, Amikacin and Carbapenem groups (IPM \& MRP) which are with $\beta$-lactamases inhibitors are the main drugs to treat multidrug resistant Pseudomonas aeruginosa as they are showing highest sensitivity. Confirmation of AmpC production supports susceptibility information, permitting Carbapenems to be reserved for complicated infections.

This may allow selection of a targeted narrow spectrum antibiotic rather than those with broad spectrum activity, minimizing the risk of selecting for, or promoting the development of resistance.

Carbapenems are highly efficacious infighting these $\beta$ - lactamase producing bacteria. Carbapenems, due to their stability to hydrolysis by most $\beta$-lactamases enzyme, have been the drugs of choice for treatment of infections caused by penicillin-resistant or cephalosporin resistant gram-negative bacterium infections.

We recommend early detection of AmpC $\beta$ lactamases production to identify the resistance of $P$. aeruginosa. The present study initially steps towards controlling the spread of MDR (Multi drug resistance).

It is the need of the hour that antibiotic policies should be formulated \& rationale use of drugs should be implemented to resist and overcome this emerging problem. Every effort should be made to prevent spread of resistant organisms.

\section{Acknowledgement}

This research has been supported by dept. of Microbiology, Sarojini Naidu Medical College, Agra (U.P), India.

\section{References}

1. Babay H A H. Antimicrobial Resistance among Clinical Isolates of Pseudomonas aeruginosa from patients in a Teaching Hospital, Riyadh, Saudi Arabia, 2001-2005. J Infect. Dis 2007; 60:123-5.

2. C. Ergin, G. Mutlu. Clinical distribution and antibiotic resistance of Pseudomonas species. Eastern Journal of Medicine 1999; 4:65-9.

3. Jacoby GA. AmpC $\beta$-lactamases. Clin Microbiol Rev. 2009; 22:161-82.

4. Thomson KS. Extended-spectrumAmpC $\beta$-lactamases, AmpC, and Carbapenemase issues. J Clin Microbiol. 2010; 48:1019-25.

5. Lister PD, Wolter DJ, HansonND. Antibacterial resistant Pseudomonas aeruginosa: clinical impact and complex regulation of chromosomally encoded resistance mechanisms. Clin Microbiol Rev. 2009; 24:582-610.

6. Sundin S. Hidden $\beta$-lactamases in Enterobacteriaceae-dropping the extra disks for detection, Part 2. Clinical Microbiology Newsletter. 2009; 31:4752.

7. Pai H, Kang CI, Byeon JH, et al., Epidemiology and clinical features of bloodstream infections caused by AmpC-type- $\beta$-lactamases-producing Klebsiella pneumonia. Antimicrob Agents Chemother 2004; 48:3720-8.

8. Sanders WE Jr., Sanders CC. Enterobacter spp.: pathogens poised to 
flourish at the turn of the century. Clin Microbiol Rev 1997; 10:220-41.

9. Neu HC. Safety of cefepime: a new extended-spectrum parental cephalosporin. Am J Med 1996; 100(6A):68S-75.

10. Food and Drug Administration. Information for Healthcare Professionals: Cefepime (marketed as Maxipime)

11. Neu HC, Chin NX, Jules K, Labthavikul P. The activity of BMY 28142 a broad spectrum $\beta$-lactamase stable cephalosporin. J Antimicrob Chemother 1986; 17:441-52.

12. Sanders CC. Cefepime: the next generation? Clin Infect Dis 1993; 17:369-79.

13. Upadhyay S, Sen MR, Bhattacharjee A (2010). Presence of different betalactamase classes among clinical isolates of Pseudomonas aeruginosa expressing AmpC beta-lactamase enzyme. J. Infect. Dev. Ctries. 4(4):239242.

14. Shoorashetty RM, Nagarathnamma T, Prathibha J (2011). Comparison of the boronic acid disc potentiation test and cefepime-clavulanic acid method for the detection of ESBL among AmpCproducing Enterobacteriaceae. Indian J. Med. Microbiol. 29(3):297-301.

15. Pollack M. Pseudomonas aeruginosa. In: Mandell GL, Bennett JE, Dolin R, eds. Principles and practice of infectious diseases. Vol. 2. Philadelphia: Churchill Livingstone, 2000:2310-35.

16. National Nosocomial Infections Surveillance (NNIS) system report: data summary from October 1986-April 1998, issued June 1998. Am J Infect Control 1998; 26:522-33.

17. Arruda EA, Marinho IS, Boulos M, et al., Nosocomial infections caused by multi resistant Pseudomonas aeruginosa. Infect Control Hosp. Epidemiol., 1999; 20:620-3.

18. Rodrigues C, Joshi $\mathrm{P}$, Jani $\mathrm{S}$, et al., Detection of $\beta$-lactamases in nosocomial gram negative clinical isolates. Indian J Medical Microbiology 2004; 22: 247-250.

19. Bhattacharjee A, Anupurba S, Gaur A, et al., Prevalence of inducible AmpC $\beta$ lactamase and metallo $\beta$-lactamasesproducing Pseudomonas aeruginosa in a tertiary care hospital in northern India. IJMM 2008; 26: 89-90.

20. Bouza E, Garcia-Gorrote F, Cercenado E, Marin M, Diaz MS. Pseudomonas aeruginosa: a survey of resistance in 136 hospitals in Spain. The Spanish Pseudomonas aeruginosa Study Group. Antimicrobial Agents Chemother 43: 981-2, 1999.

21. Bonfiglio G, Carciotto V, Russo G et al., Antibiotic resistance in Pseudomonas aeruginosa: An Italian survey. Antimicrob Chemother., 41: 307-10, 1998.

22. Rotimi VO, al-Sweih NA, Feteih J. The prevalence and antibiotic susceptibility pattern of gram-negative bacteria isolates in two ICUs in Saudi Arabia and Kuwait. Diagn Microbiol Infect Dis 30: 53-9, 1998.

23. Centers for Disease Control and Prevention. National Nosocomial Infections Surveillance (NNIS) system report, data summary from January 1990-May 1999. Am J Infect Control 27: 520-32, 1999.

24. Pfaller MA, Jones RN. MYSTIC (meropenem yearly susceptibility test information collection) results from the Americas: resistance implications in the treatment of serious infections. $\mathrm{J}$ Antimicrob Chemother 46: 25-37, 2000.

25. Jacoby GA. AmpC $\beta$-lactamases. Clin Microbiol Rev 2009; 22: 161-82. 
26. Rand KH, Turner B, Seifert H, Hansen C, Johnson JA, Zimmer A. Clinical laboratory detection of AmpC $\beta$ lactamase: does it affect patient outcome? Am J Clin Pathol 2011; 135: 572-6.

27. Pai $\mathrm{H}$, King $\mathrm{CI}$, Byeon $\mathrm{JH}$, et al., Epidemiology and clinical features of bloodstream infections caused by AmpC-type- $\beta$-lactamase-producing $K$. pneumoniae. Antimicrob Agents Chemother 2004; 48: 3720-8.

28. HarrisPN, FergusonJK. Antibiotic therapy for inducible AmpC $\beta$ lactamase-producing gram-negative bacilli: what are the alternatives to carbapenems, quinolones and aminoglycosides? Int $\mathrm{J}$ Antimicrob Agents 2012; 40:297-305.
29. Livemore DM, Brown DF, Quinn JP, Carmeli Y, Paterson DL, Yu VL. Should third generation cephalosporins be avoided against AmpC-inducible Enterobacteriaceae? Clin Microbiol Infect 2004; 10: 84-5.

30. Sanders WE Jr., Sanders CC. Enterobacter spp.: pathogens poised to flourish at the turn of the century. Clin Microbiol Rev 1997; 10:220-41.

31. Pfallar MA, Jones RN, Marshall SA, et al., Inducible ampC $\beta$-lactamase producing gram-negative bacilli from blood stream infections: frequency, antimicrobial susceptibility, and molecular epidemiology in a national surveillance program (SCOPE). Diagn Microbial Infect Dis 1997; 28: 211-9.

\section{How to cite this article:}

Arti Agrawal, Namita Srivastava, Dharmendra Kumar, Rashmi and Neha Bhati. 2017. AmpC $\beta$-lactamase Production in Pseudomonas aeruginosa: A Threat. Int.J.Curr.Microbiol.App.Sci. 6(12): 2125-2133. doi: https://doi.org/10.20546/ijcmas.2017.612.243 